\title{
Frame Aggregation-based Power-Saving Scheduling Algorithm for Broadband Wireless Networks
}

\author{
Wen-Jiunn Liu and Kai-Ten Feng \\ Department of Electrical Engineering \\ National Chiao Tung University \\ Hsinchu, Taiwan \\ jiunn.cm94g@nctu.edu.tw and ktfeng@mail.nctu.edu.tw
}

\begin{abstract}
The limitation on the battery lifetime has been a critical issue for the advancement of mobile computing. Different types of power-saving techniques have been proposed in various fields. In order to provide feasible energy-conserving mechanisms for the mobile subscriber stations (MSSs), three power-saving types have been proposed for the IEEE 802.16e broadband wireless networks. However, these power-saving types are primarily targeting for the cases with a single connection between the base station (BS) and the MSS. With the existence of multiple connections, the power efficiency obtained by adopting the conventional scheduling algorithm can be severely degraded. In this paper, with the consideration of the multiple connections and their quality-of-service $(\mathrm{QoS})$ constraints, a frame aggregationbased power-saving scheduling (FAPS) algorithm is proposed to enhance the power efficiency by aggregating multiple underutilized frames into fully-utilized ones. The performance evaluation is conducted and compared via the simulations. Simulation results show that the sleep frame ratio (i.e., a power efficiency metric) of the proposed FAPS algorithm outperforms the baseline protocols with tolerable delay.
\end{abstract}

Keywords: Power-saving, scheduling, quality of service, multiple connections, frame aggregation, broadband wireless network.

\section{INTRODUCTION}

There are significant amounts of research work [1] [2] [3] focusing on the energy-saving issues for battery-powered mobile devices in the broadband wireless networks (BWNs), such as the IEEE 802.16e standard [4]. Different types of energy efficient algorithms have been studied in [1] for generic central-controlled wireless data networks. Based on the IEEE 802.11 power-saving mechanism, several energy conservation schemes have been proposed in both centralized [2] and decentralized [3] manners. However, these techniques are not designed and intended to satisfy the requirements as defined in the IEEE 802.16e standard. In recent research studies, the performance analysis of the IEEE 802.16 e power-saving types are investigated. Most of the work concentrate on constructing the analytical models for power-saving class of type I [5] [6] [7] [8] [9]; while the enhanced model as proposed in [10] switches the power saving class between type I and II according to the network traffic. A longest virtual burst first (LVBF) scheduling algorithm has been proposed in [11],

\footnotetext{
${ }^{1}$ This work was in part funded by the Aiming for the Top University and Elite Research Center Development Plan, NSC 96-2221-E-009-016, the MediaTek research center at National Chiao Tung University, and the Universal Scientific Industrial (USI) Co., Taiwan.
}

which considers both the energy conservation and resource allocation between the BS and multiple MSSs. Nevertheless, these analytical results and scheduling schemes only consider a single connection between the BS and each MSS, i.e., a single connection is assigned to each MSS. In view of multiconnection scenarios, the periodic on-off scheme (PS) and the aperiodic on-off scheme (AS) [12] are therefore proposed. The objective of the PS scheme is to provide a scheduling algorithm with periodic sleep and listen intervals, which can elongate the total sleep intervals. However, since connections may have aperiodic traffic pattern, the PS scheme with periodic pattern is not ideal to accommodate these traffics in terms of the power efficiency. For further enhancement, the AS scheme with aperiodic sleep and listen intervals is therefore suggested. According to the delay constraint of each connection, the connection-oriented AS scheme schedules each connection from the one with the smallest delay constraint (i.e., the connection with tight QoS delay first). For each packet in the connection, the AS scheme delay the packet as much as possible in order to acquire the chance of aggregating with other packets, which can improve the power efficiency.

Instead of the connection-oriented method, a packet-level frame aggregation-based power-saving scheduling (FAPS) algorithm is proposed in this paper with the consideration of the multiple connections and their QoS constraints. The FAPS scheme consists of two procedures, including the frame aggregation procedure and the backward adjustment procedure. The frame aggregation procedure acts as the default routine of maximizing the number of sleep frames by aggregating multiple under-utilized frames into fully-utilized ones; while the backward adjustment procedure is utilized when the frame aggregation procedure encounters procedure exceptions. The performance evaluation is conducted and compared via the simulations. Simulation results show that the power efficiency of the proposed FAPS algorithm outperforms the baseline protocols with tolerable delay.

The rest of this paper is organized as follows. The targeted problem and the corresponding system model are formulated in Section II. Section III explains the proposed FAPS algorithm; while the performance evaluation of the proposed FAPS scheme is conducted in Section IV. Section V draws the conclusions and summarizes the contributions. 


\section{Problem Formulation}

On account of the inefficiency and inflexibility caused by the single connection-based IEEE 802.16e sleep mode operation, it is motivated that a feasible scheduling algorithm should be proposed to enhance the efficiency of the power scheduling under the scenarios of multiple connections. In order to model the combined effects of the multiple connections with QoS delay constraints, a packet-based modeling technique is suggested since all the connections can be divided into data packets respectively. The proposed grant space (GS) is employed as the QoS data packet model to represent each QoS data burst of connections, which is defined as in Definition 1.

Definition 1 (grant space). Given a frame $s_{i}$ with a prescheduled grant for a data burst, a grant space $G_{i}\left(s_{i}, g_{i}, t_{i}\right)$ is defined as the adjacent frames ranging from $s_{i}$ to $t_{i}=$ $s_{i}+D_{i}-1$, where $D_{i}$ is the maximum QoS delay constraint for this data burst. The frames $s_{i}$ and $t_{i}$ are respectively called the start and the termination for this grant space, and the frame $g_{i}$ is the frame for containing the data burst.

The left upper plot of Fig. 1 illustrates the grant space $G_{i}\left(s_{i}, g_{i}, t_{i}\right)$ with the delay constraint $D_{i}$. In order to maintain the QoS requirement, the data burst should be scheduled within the frame $g_{i}$, where $s_{i} \leq g_{i} \leq t_{i}$ (i.e., between the start and the termination frames). For example, the grant space of the data burst $a$ is $G_{a}\left(s_{a}, g_{a}, t_{a}\right)$, where $s_{a}=1, g_{a}=1$, $t_{a}=5$, and $D_{a}=5 \cdot T_{f}$. Thanks to the adoption of the grant spaces, the whole system can therefore be modeled by multiple grant spaces acquired from multiple connections. As shown in Fig. 1, there are nine connections and each connection consists of multiple data bursts with their maximum QoS delay constraints. Based on Definition 1, each data burst with its delay constraint can be modeled as a grant space. When completing the representation of data bursts, the MSS listen and sleep frames can be determined by the arrangement of each data burst. For example, the frame 1 of the MSS should be a listen frame since the first data packet $a$ is scheduled within this frame; while the frame 6 is a sleep frame because of no scheduled data packet. Finally, all MSS listen frames are represented as the grey frames; while the sleep frames can be identified as the white ones in Fig. 1. It is noted that each MSS frame can only contain $F_{\max }$ data bursts at most since the accommodation of a frame is usually limited. After the description of the system model, our targeted packet-based power-saving scheduling (PPS) problem is how to arrange the data bursts within the grant spaces in order to maximize the power efficiency (i.e., the number of sleep frames)?

\section{Proposed Frame Aggregation-BAsed Power-SAVING SCHEDUling (FAPS) Algorithm}

In order to solve our PPS problem, a frame aggregationbased power-saving scheduling (FAPS) algorithm is proposed. With the proper arrangement of data bursts, the proposed FAPS algorithm can enhance the power efficiency under the constraint of the pre-specified QoS delay requirement. The FAPS

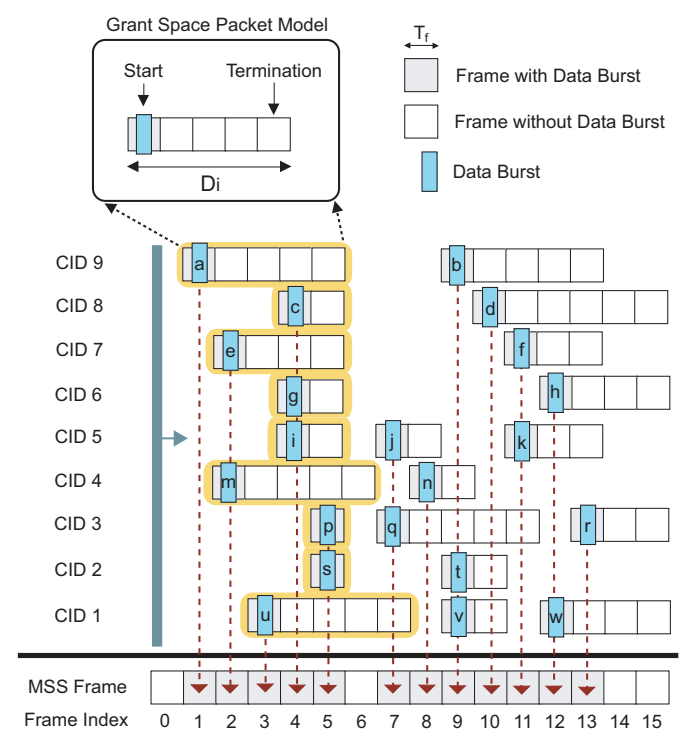

Fig. 1. The grant space and the system model.

scheme consists of two procedures, including the frame aggregation procedure and the backward adjustment procedure. The frame aggregation procedure acts as the default routine of maximizing the number of sleep frames in the proposed FAPS scheme; while the backward adjustment procedure is utilized when the frame aggregation procedure encounters procedure exceptions. The two procedures are described as follows.

\section{A. Frame Aggregation Procedure}

The objective of the frame aggregation procedure is to acquire more sleep frames by aggregating some under-utilized listen frames into a fully-utilized one. For example, the maximum accommodation $F_{\max }$ of an MSS frame is assumed to be 3. As shown in Fig. 1, the two under-utilized listen frame (i.e., the frame 7 and the frame 8 ) can be aggregated into the frame 8 together since the three corresponding data bursts (i.e., the bursts $j$ and $q$ in the frame 7 and the burst $n$ within the frame 8) can be scheduled at the frame 8 without breaking the QoS delay constraints specified in their grant spaces. The additional sleep frame at the frame 7 can therefore be acquired, which improves the power efficiency of the system. The proposed frame aggregation procedure is described as follows:

1) Construction of Stuck Groups: It is noted that if a data burst $i$ can be delayed, there is more chance to aggregate with other data burst, gaining more sleep frames. However, in order to meet the QoS delay constraint, the data burst $i$ must be scheduled before the termination $t_{i}$ specified within the grant space $G_{i}\left(s_{i}, g_{i}, t_{i}\right)$. Therefore, the first step of our procedure is to delay all data bursts from left to right before some data burst violates its delay constraint. For example, as shown in Fig. 1, the set of data bursts $\mathbf{D}=\{a, c, e, g, i, m, p, s, u\}$ (i.e., their grant spaces are with yellow background color) can be delayed to the frame 5 . However, these data bursts $a, c, e$, $g, i, p$, and $s$ will violate their delay constraints if continuing to delay them to the frame 6 . This action can be viewed as 


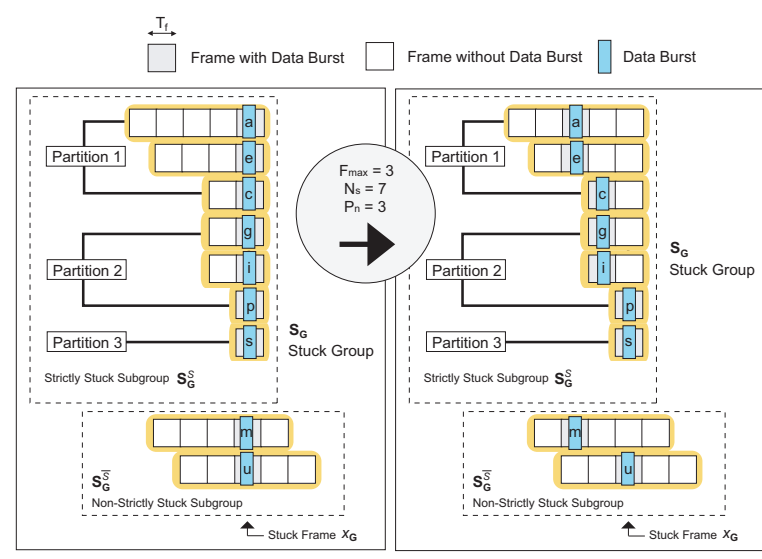

Fig. 2. The proposed frame aggregation procedure.

using a windscreen wiper, i.e., the solid bar at the frame 0 in Fig. 1. The windscreen wiper will be stuck at the frame $x_{\mathbf{G}}=5$ since hazards are encountered, i.e., the data bursts $a$, $c, e, g, i, p$, and $s$. Therefore, the corresponding unscheduled grant spaces stuck at the frame $x_{\mathrm{G}}$ (i.e., the grant spaces with yellow background for the set $\mathbf{D}$ ) can be formally defined as the stuck group $\mathbf{S}_{\mathbf{G}}$. In a stuck group, there may exist some data bursts in the grant spaces, which still can be delayed. As shown in Fig. 1, the data bursts $m$ and $u$ with their grant spaces specified in the stuck group $\mathbf{S}_{\mathrm{G}}$ can be delayed to the frame 6 and the frame 7 respectively. These grant spaces which can be further delayed are categorized as the non-strictly stuck subgroup $\mathbf{S}_{\mathbf{G}}^{\bar{S}}$; while the remaining grant spaces in $\mathbf{S}_{\mathbf{G}}$ are called the strictly stuck subgroup $\mathbf{S}_{\mathbf{G}}^{S}$.

2) Backward Push Mechanism: As shown in the left plot of Fig. 2, the stuck group $\mathbf{S}_{\mathbf{G}}$ obtained from the data burst set $\mathbf{D}=\{a, c, e, g, i, m, p, s, u\}$ in Fig. 1 is sorted by the termination and by the start if having the same termination. It is also separated into the strictly stuck subgroup $\mathbf{S}_{\mathbf{G}}^{S}$ and the non-strictly stuck subgroup $\mathbf{S}_{\mathbf{G}}^{\bar{S}}$. It can be observed that all data bursts in $\mathbf{D}$ will be stuck at the stuck frame $x_{\mathbf{G}}$. However, it is impossible to schedule all of them into the frame $x_{\mathbf{G}}$ if the maximum accommodation $F_{\max }$ of an MSS frame is less than the size of $\mathbf{S}_{\mathrm{G}}$. Therefore, in the second step of our proposed frame aggregation procedure, the maximum frame accommodation of $F_{\max }$ should be considered. In addition, the scheduling of data bursts specified in the grant spaces of $\mathbf{S}_{\mathbf{G}}^{S}$ should be done prior to $\mathbf{S}_{\mathbf{G}}^{\bar{S}}$ since all data bursts in $\mathbf{S}_{\mathbf{G}}^{S}$ must be scheduled before the stuck frame $x_{\mathbf{G}}$. The arrangement of the data bursts specified in $\mathbf{S}_{\mathbf{G}}^{S}$ can be done as follows: Let $N_{s}$ be the number of grant spaces in $\mathbf{S}_{\mathbf{G}}^{S}$, and $P_{n}=\left\lceil N_{s} / F_{\max }\right\rceil$ be the number of partitions utilized to separate $\mathbf{S}_{\mathrm{G}}^{S}$ from the first grant space. It is noted that each partition of $\mathbf{S}_{\mathbf{G}}^{S}$ may have $F_{\max }$ data bursts at most so as to meet the requirement of the frame accommodation. Finally, referenced at the stuck frame $x_{\mathbf{G}}$, let the data bursts of each partition $i$ move back $P_{n}-i$ frames gradually if possible. Otherwise, stop moving at the frame unable to proceed. It completes the arrangement of the data bursts specified in $\mathbf{S}_{\mathbf{G}}^{S}$. For example, as shown in the right

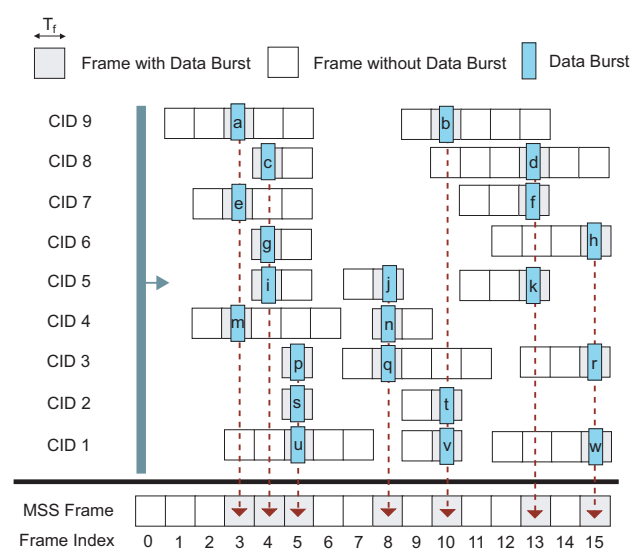

Fig. 3. The scheduling result of the proposed frame aggregation procedure.

plot of Fig. 2, the maximum frame accommodation is $F_{\max }=$ 3 , the number of grant spaces in $\mathbf{S}_{\mathbf{G}}^{S}$ is $N_{s}=7$, and the number of partitions is $P_{n}=\left\lceil N_{s} / F_{\max }\right\rceil=3$. The data bursts in the partition 1 should be moved back $P_{n}-1=2$ frames. However, the data burst $c$ will simply be proceeded back one frame since it is limited by the grant space $G_{c}\left(s_{c}, g_{c}, t_{c}\right)$. In the partition 2 , the same situation can also be found at the data burst $p$ which is constrained by the grant space $G_{p}\left(s_{p}, g_{p}, t_{p}\right)$. Finally, the data bursts $a$ and $e$ will be scheduled in the same frame. The bursts $c, g$, and $i$ can also be aggregated; while the stuck frame can accommodate the remaining data bursts $p$ and $s$.

3) Packet Padding Mechanism: On the other hand, the scheduling of the non-strictly stuck subgroup $\mathbf{S}_{\mathbf{G}}^{\bar{S}}$ can be considered as a data burst stuff for some under-utilized listen frame to improve the power efficiency. Therefore, the data bursts specified in $\mathbf{S}_{\mathbf{G}}^{\bar{S}}$ can be scheduled with those in $\mathbf{S}_{\mathbf{G}}^{S}$ as follows: Starting from the left, for a under-utilized listen frame $\delta$, find a proper number of grant spaces in $\mathbf{S}_{\mathbf{G}}^{\bar{S}}$ whose data burst can be scheduled in the frame $\delta$. As shown in the right plot of Fig. 2, the first under-utilized listen frame is the frame with data bursts $a$ and $e$, and the second one is the frame with data bursts $p$ and $s$. Based the aforementioned technique, the data burst $m$ will be arranged together with the data bursts $a$ and $e$; while the burst $u$ will be accommodated with the bursts $p$ and $s$, leading to the fully-utilized listen frames. Finally, if there still remain some data bursts specified in the grant spaces of $\mathbf{S}_{\mathbf{G}}^{\bar{S}}$, these data bursts should be considered with the other unscheduled grant spaces as the new input grant spaces for the next round of the frame aggregation procedure. It can be perceived that the data burst set $\mathbf{D}=\{a, c, e, g, i, m, p$, $s, u\}$ as in Fig. 1 can be successfully scheduled within three listen frames, which is smaller than the original five listen frames. Furthermore, Fig. 3 shows the scheduling result of the complete power-saving system exemplified in Fig. 1 after five rounds of execution of the frame aggregation procedure. It can be observed that the required listen frames is decreased from the original 12 to the current 7 listen frames. The enhancement of power efficiency can be obtained in the proposed frame aggregation procedure. 


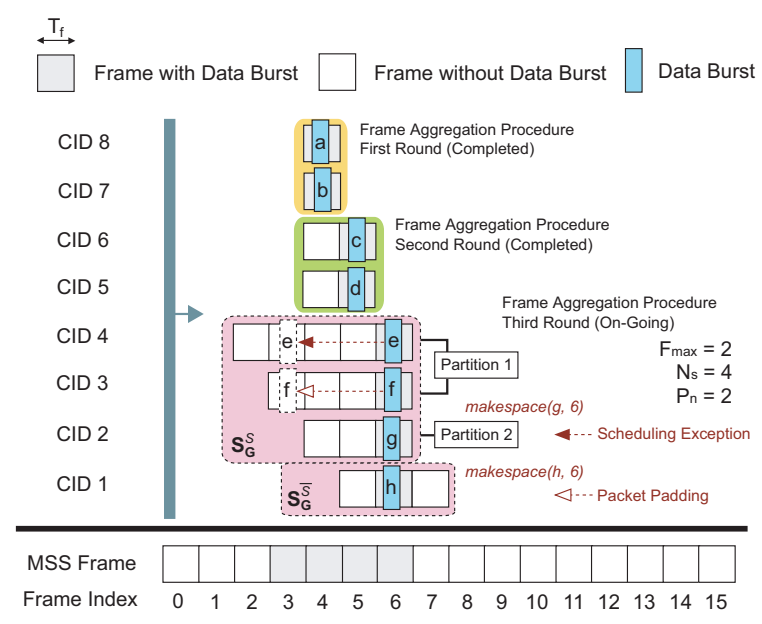

Fig. 4. The proposed backward adjustment procedure for the scheduling exceptions of the frame aggregation procedure.

\section{B. Backward Adjustment Procedure}

The backward adjustment procedure is utilized to solve the scheduling exceptions encountered by the aforementioned frame aggregation procedure. As shown in Fig. 4, there are eight data bursts (i.e., $a, b, c, d, e, f, g$, and $h$ ) from the eight connections, and the maximum accommodation $F_{\max }$ of an MSS frame is assumed to be 2 . In the first round of the execution of the frame aggregation procedure, the data bursts $a$ and $b$ is completely scheduled. Moreover, the data bursts $c$ and $d$ are also successfully aggregated in the second round without exceptions. In the third round of execution, there are four unscheduled grant spaces for the data bursts $e, f, g$, and $h$, which are separated into the strictly and non-strictly stuck subgroups $\mathbf{S}_{\mathbf{G}}^{S}$ and $\mathbf{S}_{\mathbf{G}}^{\bar{S}}$. Based on the frame aggregation procedure, the grant spaces of $\mathbf{S}_{\mathbf{G}}^{S}$ will be divided into multiple partitions. For example, as shown in Fig. 4, the partition 1 consisting of data bursts $e$ and $f$ and the partition 2 containing $g$ will be constructed. In the partition 1 , each data burst should be moved backward $P_{n}-1=\left\lceil N_{s} / F_{\max }\right\rceil-1=1$ frame. Nevertheless, it is unable to let them backward since the frame 5 is full of data bursts, i.e., the data bursts $c$ and $d$. Fortunately, the data bursts $e$ and $f$ still can be placed at the frame 6 . On the other hand, for the data burst $g$ specified in the partition 2, they should be scheduled at the frame 6 . However, the exception of the proposed frame aggregation procedure occurs since the frame 6 has the maximum number $F_{\max }=2$ of data bursts $e$ and $f$ and the frame 6 is the only choice for the partition 2 .

The proposed backward adjustment procedure is described as follows: Given a data burst $\lambda$ and the targeted frame $\theta$, a function makespace $(\lambda, \theta)$ is utilized to make a space for $\lambda$ at the frame $\theta$ by the backward recursive data burst movement. If $\lambda$ can be scheduled in $\theta$, the function makespace will return true. Consequently, the data burst $\lambda$ will be scheduled in the targeted frame $\theta$, solving the exception resulting from the frame aggregation procedure. If $\lambda$ can not be scheduled into the frame $\theta$, let $\xi$ be the data burst in $\theta$ whose grant space $G_{\xi}\left(s_{\xi}, g_{\xi}, t_{\xi}\right)$ has the smallest start frame. Subsequently, based on the same function makespace, try to backward search the non-saturated frame $\pi$ ranging from the frame $(\theta-1)$ to the start frame $s_{\xi}$. If the frame $\pi$ can be found, schedule the data burst $\xi$ into this frame $\pi$. Finally, put the unscheduled data burst $\lambda$ into the frame $\theta$, completing the backward adjustment procedure for scheduling the data burst $\lambda$.

As shown in Fig. 4, in the third round of the frame aggregation procedure, when starting to arrange the data burst $g$ in the partition 2, the data burst $g$ will encounter the exception of the frame aggregation procedure. Therefore, based on the backward adjustment procedure, the function makespace $(\lambda, \theta)$ will be invoked, where $\lambda=g$ and $\theta=6$. For the data bursts $e$ and $f$ scheduled in the frame 6 , the grant space $G_{e}\left(s_{e}, g_{e}, t_{e}\right)$ of the data burst $e$ has the smallest start (i.e., $s_{e}=2$ ). Consequently, the data burst $e$ is selected as the burst which should be moved back.

Subsequently, the function makespace $(\lambda, \theta)$ will be reconducted for the new input $\lambda=e$ and several new input $\theta$ of $\pi=\theta-1, \ldots, s_{i}$ (i.e., $\pi=5,4,3$, and 2 for each time). When the first function makespace $(e, 5)$ for scheduling the data burst $e$ is invoked, the next selected data burst which may be moved back is the burst $c$ in the frame 5 since it has the smallest start, i.e., the frame 4 . Recursively, when makespace $(c, 4)$ for arranging the data burst $c$ is invoked, the burst $a$ is also chosen as the candidate to move back. However, $a$ can not be moved back since it is at the start frame 4 of the grant space $G_{a}\left(s_{a}, g_{a}, t_{a}\right)$. Since the burst $a$ can not be moved, the data burst $c$ simply can be scheduled at the frame 5 . Moreover, since $c$ can not be moved back, the burst $e$ should not be scheduled at the frame 5 . Therefore, makespace $(e, 5)$ can not make a space for $e$ at the frame 5 .

The second function makespace $(e, 4)$ for scheduling the data burst $e$ will continually be invoked to check if the burst $e$ can be scheduled at the frame 4 . For the same reason, the burst $e$ also can not be arranged at the frame 4. Finally, the data burst $e$ will be scheduled at the frame 3 since the third function makespace $(e, 3)$ for scheduling the burst $e$ will return the value of true, representing the vacancy of the frame 3 . When the burst $e$ is placed into the frame 3 , it leaves a vacancy in the frame 6 for the data burst $g$. Therefore, $g$ can be scheduled at the frame 6 , completing the scheduling of the burst $g$. It can be observed that there is a under-utilized frame caused by the makespace function (i.e., the frame 3). In order to gain the power efficiency, the data burst $h$ in $\mathbf{S}_{\mathbf{G}}^{\bar{S}}$ will be scheduled at the frame 6 by conducting the function makespace $(h, 6)$. Thanks to the backward adjustment procedure, the third round of the frame aggregation procedure can therefore be completed.

\section{Performance Evaluation}

In this section, simulations are conducted to evaluate the performance of the proposed FAPS scheduling algorithms in comparison with the AS scheme and the original powersaving mechanism in the IEEE $802.16 \mathrm{e}$ specification. A single BS/MSS pair with multiple connections are considered as the simulation scenario. 100 frames with each frame duration of 5 


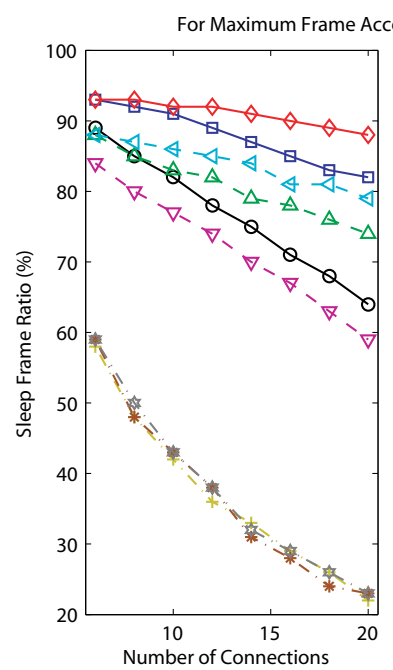

(a)

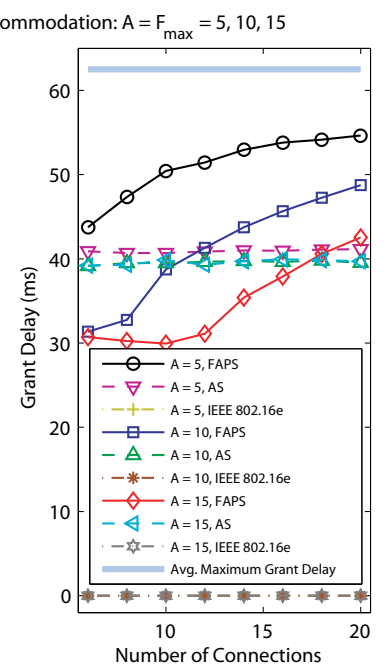

(b)
Fig. 5. The performance comparison of the proposed FAPS, AS, and IEEE 802.16 e protocols.

ms are utilized in the simulation. The default settings of each connection are listed as follows: The connection period (i.e., the number of frames between each two consecutive packets) is 10; while the delay constraint is ranging from $50 \mathrm{~ms}$ to 75 ms. Two performance metrics are adopted for performance comparison: 1) the sleep frame ratio: the number of sleep frames divided by the total frames, 2) the grant delay: the average value of $\left(g_{i}-s_{i}\right)$ for each grant space $G_{i}\left(s_{i}, g_{i}, t_{i}\right)$.

The performance curves of the sleep frame ratio under different maximum frame accommodations are shown in Fig. 5(a). The curves of IEEE $802.16 \mathrm{e}$ are almost the same under $F_{\max }$ of 5,10 , and 15 since it does not rearrange the data packets and just schedule the packets at the original start frames specified in their grant spaces. On the other hand, the performance of the sleep frame ratio of the proposed FAPS protocol outperforms the other connection-oriented schemes under each $F_{\max }$ since the FAPS protocol can effectively rearrange the data packets in the packet level. It is noted that when the number of connections is increased, the sleep frame ratio is decreased since more frames should be awake to serve the increased number of packets. It is also noted that when the maximum frame accommodation becomes larger, the sleep frame ratio is increased since less frames should be consumed to contain the packets.

Fig. 5(b) shows the performance curves of the grant delay under different maximum frame accommodations. The grant delay performance of the IEEE $802.16 \mathrm{e}$ should be zero since it does not delay its packets. In the curves of the FAPS and the AS schemes, when the maximum frame accommodation becomes smaller, the more grant delay should be generated since more packets should be delayed to seek the proper position to accommodate themselves. The curves of the AS scheme maintain almost a constant trend since the AS scheme is a connection-oriented scheme for conducting scheduling from the connection with the smallest delay constraint, which dominates the performance curves. In general, the grant delay of the FAPS scheme should be larger than that of the AS algorithm since the FAPS scheme has higher performance in the sleep frame ratio. However, in some cases, the curves of the FAPS scheme will violate this general thought because the connection-oriented AS scheme is dominated by the connection with the smallest delay, while the FAPS algorithm is a fully packet-level method. The FAPS can even find a better way with smaller delay to aggregate packets. In the end, it can be observed that all curves are below the average maximum grant delay, which maintains the QoS delay constraint requirements.

\section{CONCLUSION}

In this paper, with the consideration of the multiple connections and their QoS constraints, a packet-level frame aggregation-based power-saving scheduling (FAPS) algorithm is proposed to maximize the number of total sleep frames by frame aggregation techniques. Simulation studies show that the power efficiency of the proposed FAPS algorithm outperforms the other baseline protocols with tolerable delay.

\section{REFERENCES}

[1] J.A. Stine and Gustavo de Veciana, "Improving Energy Efficiency of Centrally Controlled Wireless Data Networks," ACM/Baltzer Wireless Networks, vol. 8, pp. 681-700, Nov. 2002.

[2] R. Krashinsky and H. Balakrishnan, "Minimizing Energy for Wireless Web Access with Bounded Slow-down," in Proc. ACM/IEEE Int. Conf. on Mobile Computing and Networking (MobiCom), Sept. 2002, pp. 119130.

[3] K.T. Feng and K.H. Chou, "Intelligent Router-Assisted Power Saving Medium Access Control for Mobile Ad Hoc Networks," in Proc. IEEE Vehicular Technology Conference (VTC), May 2006, pp. 304-308.

[4] IEEE 802.16 WG, IEEE Standard 802.16e-2005: Part 16: Air Interference for Fixed Broadband Wireless Access Systems, Amendment 2: Physical and Medium Access Control Layers for Combined Fixed and Mobile Operation in Licensed Bands and Corrigendum 1, IEEE Standards Association Std., 2006.

[5] Y. Xiao, "Energy Saving Mechanism in the IEEE 802.16e Wireless MAN," IEEE Commun. Lett., vol. 9, pp. 595-597, Jul. 2005.

[6] K. Han and S. Choi, "Performance Analysis of Sleep Mode Operation in IEEE 802.16e Mobile Broadband Wireless Access Systems," in Proc. IEEE Vehicular Technology Conference (VTC), May 2006, pp. 11411145.

[7] Y. Park and G.U. Hwang, "Performance Modelling and Analysis of the Sleep-Mode in IEEE802.16e WMAN," in Proc. IEEE Vehicular Technology Conference (VTC), Apr. 2007, pp. 2801-2806.

[8] Y. Zhang, "Performance Modeling of Energy Management Mechanism in IEEE 802.16e Mobile WiMAX," in Proc. IEEE Wireless Communications and Networking Conference (WCNC), Mar. 2007, pp. 3205-3209.

[9] Y. Xiao, "Performance Analysis of an Energy Saving Mechanism in the IEEE 802.16e Wireless MAN," in Proc. Consumer Communications and Networking Conference (CCNC), Jan. 2006, pp. 406-410.

[10] L. Kong and D.H.K. Tsang, "Optimal Selection of Power Saving Classes in IEEE 802.16e," in Proc. IEEE Wireless Communications and Networking Conference (WCNC), Mar. 2007, pp. 1836-1841.

[11] J. Shi, G. Fang, Y. Sun, J. Zhou, Z. Li, and E. Dutkiewicz, "Improving Mobile Station Energy Efficiency in IEEE 802.16e WMAN by Burst Scheduling," in Proc. IEEE Global Communications Conference (GLOBECOM), Nov. 2006, pp. 1-5.

[12] Y.L. Chen and S.L. Tsao, "Energy-efficient Sleep-mode Operations for Broadband Wireless Access Systems," in Proc. IEEE Vehicular Technology Conference (VTC), Sept. 2006, pp. 1-5. 